\title{
Estimation of Reversed Flow in Long Pipeline Based on Axial Vibration Model of Dense Paste
}

\author{
Fuyan Lyu, ${ }^{1}$ Minjun Zhang, ${ }^{1}$ Yuanyuan Qu, ${ }^{1}$ Jie Gao, ${ }^{2}$ Xuankai Jia, \\ Xuedi Hao, ${ }^{1}$ and Miao $\mathrm{Wu}^{1}$ \\ ${ }^{1}$ Mechanical Electronic and Information Engineering, China University of Mining and Technology, Beijing 100083, China \\ ${ }^{2}$ College of Electronic Communication and Physics, Shandong University of Science and Technology, Qingdao, Shandong 266590, China
}

Correspondence should be addressed to Fuyan Lyu; fuyanjiayou2012@sina.com

Received 19 October 2015; Revised 9 February 2016; Accepted 14 February 2016

Academic Editor: Radoslaw Zimroz

Copyright (C) 2016 Fuyan Lyu et al. This is an open access article distributed under the Creative Commons Attribution License, which permits unrestricted use, distribution, and reproduction in any medium, provided the original work is properly cited.

Intense axial vibration of the paste and the reversed flow make damage to the transportation system and lag the transport progress. This paper analyzed the axial vibration of the paste by building the vibration model according to the working situations of the system. The amount of the reversed flow share is then estimated based on the formed axial vibration model. The estimation of the reversed flow is carried out by counting the amount of the decompressed paste in certain time, which is being relative to the displacement in a single process of the vibration in numerical. The estimation of the reversed flow share of coal slime paste under certain transportation conditions was given and compared with the result based on the wave velocity method. It is evident that the introduction of axial vibration model into the study of the pipeline transportation system is feasible and reasonable, which also supplements the theoretical foundation for the analysis of dense paste vibrations in pipeline transportation system and its impacts on the system.

\section{Introduction}

The concerns on the use of the high density, thickened tailings in mining industry have been enhanced significantly recently. These tailings and other similar wastes or byproducts in industry are referred to as "dense paste" in this paper to present a sort of material that consists of high solid contents and thin grain, showing high viscosity with a state of somehow between solid and fluid. This sort of material, namely, the dense paste, is widely dealt with in mining zones in Australia, South Africa, Canada, and so forth; say the mining particulate solid wastes are reused for surface and underground filling under the requirements of water conservation, cutting the risk of catastrophic failure, and potentially fascinating the final closure of the mining or even reducing the overall cost of the mining projects [1-4].

Typical dense pastes are the coal tailing from coal preparation, red mud from aluminum industry, calcium carbide sludge from acetylene production, sewage sludge from municipal waste water treatment, and so on [5]. The annual outputs of dense pastes are enormous around the world. People keep searching for efficient disposals against this kind of wastes considering energy saving and environment protection. In China, approximately 63.9 million tons of coal slime had been sent to CFB (Circulating Fluidized Bed) boilers as a partial substitution of coal fuel from 2002 to 2008 , resulting in an economic benefit of about 10.1 billion CNY [6]. Under this background, how to transport those huge amounts of dense paste in different disposals is one of the fundamental engineering problems. Pipeline transportation system driven by pumps was introduced to this area based on its characteristics such as cleanness (avoid exposure in the open air), high efficiency, and labor saving. In the real application, the transportation distance along the pipelines is often near or over $1000 \mathrm{~m}$; the used pipelines often have the diameters up to $300 \mathrm{~mm}$; and the maximum pumping pressure in the system is up to $20 \mathrm{MPa}$ [5].

Aiming for higher efficiency, the pipeline transportation system employs duplex pump with the S-shape pipe valve. The duplex pump has two material chambers which connect 

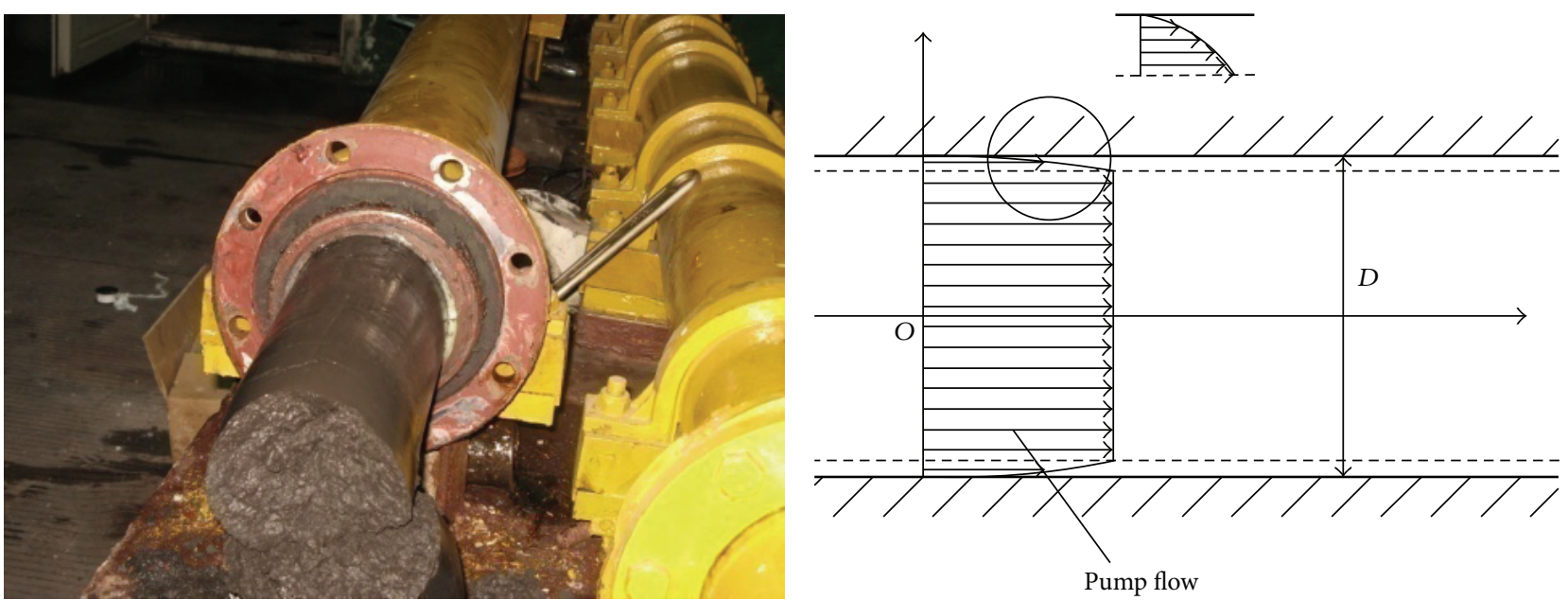

FIGURE 1: The appearance of the dense paste body in pipeline; and a thin water-rich layer forms between the paste body and inner pipe wall.

to the pipeline alternatively by switching the S-shape pipe valve between the pump and the pipeline. The working process is illustrated as follows: the piston in cylinder number 1 moves backward to inhale dense paste from the storage, while the piston in cylinder number 2 moves forward to expel the dense paste into the pipe through the S-shape pipe valve; then the valve switches; the pistons in the two cylinders then move forward and backward, respectively, to keep driving the paste flow inside the pipelines. There is a short dynamic pause going with the switch exactly. Notice that the body of dense paste in the pipeline shows viscoelasticity under pressure, which is very different from the flowing dilute slurry in normal cases. It results in the phenomenon where at the short moment when the pressure from the pump disappears the body of dense paste tries to recover and forms a force towards its two terminals.

Hao et al. [5] carried out a test from which it shows that the elastic recovery of the coal slime has an important effect on the actual flow rate. During the switch of the S-shape pipe valve, elastic recovery of the compressed paste causes part of the paste in the pipeline to flow back into the feed chamber of the pump, that is, the reversed flow. Reversed flow not only reduces the actual flow rate greatly but also causes strong axial vibration of the dense paste body and pipelines, which damages the transport equipment, especially the hopper, during the transportation $[5,7,8]$.

Reversed flow and vibration would be enhanced as the transportation distance or the pumping pressure increases. This paper aims to study the axial vibration of the body of dense paste and to analyze the amount of reversed flow. By building the axial vibration model of the dense paste according to the characters of the paste flow in long pipelines, when the driving force loses, the resulting reversed flow is predictable in volume and would be discussed based on both experiments and theoretical guidance.

In the following sections, the vibration model is derived and interpreted firstly, followed by the prediction about the reversed flow in volume ratio according to the vibration model and system working conditions. Finally, experimental observations of the reserved flow are presented to compare with the numerical estimations.

\section{Materials and Methods}

2.1. The State of the Paste When It Flows in Pipeline. The relative experiments in this paper take the coal slime, for example. Coal slime is the main tailing of coal preparation; it consists of coal, fine rocks, and clay. The original coal slurry (without mixing with other materials) is characterized by high viscosity, low fluidity, and great stickiness and is a typical non-Newtonian fluid. The solid concentration of coal slime usually ranges from $56 \%$ to $77 \%$ by weight, which was measured by Sartorius MA145 in the labs; the apparent viscosity ranges from tens to hundreds of $\mathrm{Pa} \cdot \mathrm{s}$ [5]. It behaves as a plug flow when being transported in pipes, as shown in Figure 1, with a thin water-rich layer which formed over the inner walls of the pipes during flowing. The solid concentration of the coal slime used in this paper is $59 \% \mathrm{w} / \mathrm{w}$, while the density of the coal slime is $\rho=1.50 \mathrm{~g} / \mathrm{cm}^{3}$. The yield stress of the used coal slime is $64.9 \mathrm{~Pa}$, which was tested by a vane rheometer.

\subsection{Axial Vibration Measurement Method}

2.2.1. Mechanical Model of Dense Paste in Pipeline. The dense paste behaves as non-Newtonian fluids whose state is between solid and liquid and shows viscosity and elasticity under pressure in pipes; therefore it is regarded as viscoelastic body in the mechanical model. As mentioned before that the dense paste moves in the form of a plug flow, an assumption is thereby formed where the body of dense paste in pipeline could be treated as a viscoelastic rod, as shown in Figure 2. Based on Kelvin viscoelastic rod model [7, 9-12], the force situation of the viscoelastic rod in pipeline is analyzed. The paper aims to simplify the estimated vibration of long pipeline, so the boundary conditions of the vibration model are simplified. The following assumptions were made for the formulation: 


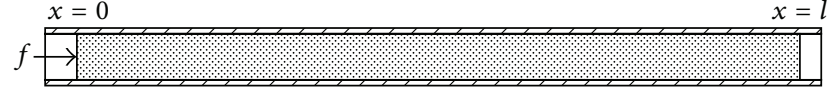

FIGURE 2: Mechanical model of the dense paste in pipeline.

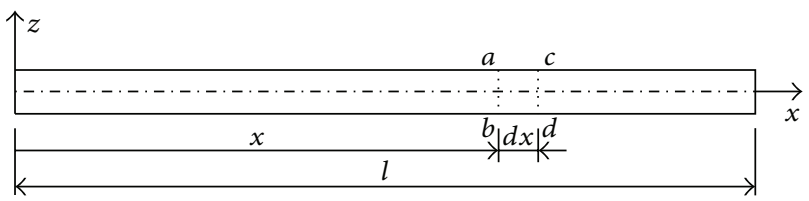

Figure 3: Geometrical diagram of paste rod.

(1) The dense paste in pipe is regarded as viscoelastic rod model, with a length of $l$ and a cross section of $A$.

(2) Material damping of dense paste is ignored and the friction between the paste and pipeline is also neglected during the vibration.

(3) The dense pastes are pumped continuously at the pressure of $f$ in experimental process.

2.2.2. Axial Vibration Analysis. In Figure 2, the left end of paste rod is next to the exit of the pump, and the right end is the exit for discharge. The dense paste is treated as Kelvin viscoelastic rod which is considered as the paralleled combination of Spring hook and Newton sticky pot. The geometrical diagram of the paste rod is shown in Figure 3. The rod locates along $x$-axis and is divided into microunits, each of which owns an axial length of $d_{x}$. The unit at the left end $x=0$ is supposed to connect with the exit of pump.

Suppose the force on the mini-element cross-sectional area on this $\operatorname{rod}$ is $F$ :

$$
\begin{aligned}
& F=\sigma A \\
& \sigma=E \varepsilon+\eta \frac{\partial \varepsilon}{\partial t},
\end{aligned}
$$

where $\sigma$ is the local stress component in $x$ direction and $A$ is the cross-sectional area of pipeline. $E$ is Young's modulus of the paste, and $\eta$ is the viscosity. $\varepsilon$ is the local strain component in $x$ direction. If $u(x, t)$ is the vibration displacement along $x$-axis at moment $t$ for the microunit located at $x$ in Figure 3, the axial strain of the unit is $\varepsilon=\partial u / \partial x$. Substituting (2) and $\varepsilon=\partial u / \partial x$ into (1) yields the expression of the force $F$ :

$$
F=E A \frac{\partial u}{\partial x}+A \eta \frac{\partial^{2} u}{\partial t \partial x} .
$$

On the other hand, according to the force balance of the microunit at locations $x$, the following equation is obtained:

$$
d F=\rho A d x \frac{\partial^{2} u}{\partial t^{2}},
$$

where $\rho$ is density of the paste. By using the derivation $d F=$ $(\partial F / \partial x) d x$ for (3) and then combining with (4), the axial vibration differential equation of a certain microunit of the paste rod can be given:

$$
E \frac{\partial^{2} u}{\partial x^{2}}+\eta \frac{\partial^{3} u}{\partial x^{2} \partial t}=\rho \frac{\partial^{2} u}{\partial t^{2}} .
$$

The axial pressure on the left end $(x=0)$ of the rod depends on the thrust of actuating pump and is set as $f$. Since the right end of the rod is open to air, the local outer pressure is zero [13]. The boundary conditions of this vibration rod are summarized by

$$
\begin{aligned}
E A\left(\frac{\partial u}{\partial x}+\tau \frac{\partial^{2} u}{\partial t \partial x}\right) & =f, \quad x=0 \\
\frac{\partial u}{\partial x}+\tau \frac{\partial^{2} u}{\partial t \partial x} & =0, \quad x=l .
\end{aligned}
$$

The initial displacement and velocity of the dense paste rod can be written in the following form:

$$
\begin{aligned}
& u(x, 0)=\varphi(x) \\
& \dot{u}(x, 0)=g(x) .
\end{aligned}
$$

During the short moment when the S-shape pipe valve switches, the pressure suddenly disappeared, which means the pump pressure turns to $f=0$; the viscoelastic rod then decompresses like a spring vibrating along the pipe and resulting in the reversed flow. Assuming that the paste rod performs free vibration when the pressure from the pump disappears, the amplitude of the vibration will reduce as the motion continues. The amount of reversed flow relates to the displacement of the paste rod during vibration that acts weaker and weaker.

According to the study of free axial vibrations, (5) can be solved using the given boundary conditions. Equation (5) can be rewritten in the following form:

$$
\frac{\partial^{2} u}{\partial x^{2}}+\tau \frac{\partial^{3} u}{\partial x^{2} \partial t}=\frac{1}{c^{2}} \frac{\partial^{2} u}{\partial t^{2}},
$$

where $\tau=\eta / E$ denotes the relaxation time of viscoelastic body; $c^{2}=E / \rho ; c$ is the pressure wave velocity. To solve (9), separation of variables is applied by setting $u(x, t)=$ $U(x) T(t)$; therefore, (9) turns out to be

$$
\frac{\ddot{U}(x)}{U(x)}=\frac{\ddot{T}(t)}{c^{2}[T(t)+\tau \dot{T}(t)]}=-\lambda^{2} .
$$

Solutions of (10) are figured out and presented in the following form:

$$
\begin{aligned}
U(x) & =C_{1} \cos \lambda x+C_{2} \sin \lambda x \\
T(t) & =e^{-\alpha t}\left[B_{1} \cos \omega t+B_{2} \sin \omega t\right] .
\end{aligned}
$$

According to (11) and (12), the displacement of certain unit on the paste rod is

$$
\begin{aligned}
u(x, t)= & e^{-\alpha t}\left[B_{1} \cos \omega t+B_{2} \sin \omega t\right] \\
& \cdot\left[C_{1} \cos \lambda x+C_{2} \sin \lambda x\right],
\end{aligned}
$$


where $B_{1}$ and $B_{2}$ and $C_{1}$ and $C_{2}$ are the undetermined coefficients determined by the initial conditions and boundary conditions, respectively; $\alpha$ is the attenuation coefficient and $\omega$ is the circular frequency of the axis vibration of the paste rod. And the related coefficients $\alpha$ and $\omega$ are defined as follows:

$$
\begin{aligned}
& \alpha=\frac{\tau \lambda^{2} c^{2}}{2} \\
& \omega=\lambda c \sqrt{1-\frac{\tau^{2} \lambda^{2} c^{2}}{4}} .
\end{aligned}
$$

When the pump stops working, the pressure on the exit of the pump $(x=0)$ changed to zero; again, (6) gives the boundary case:

$$
\frac{\partial u(0, t)}{\partial x}+\tau \frac{\partial^{2} u(0, t)}{\partial t \partial x}=0 .
$$

By taking $u(x, t)=U(x) T(t)$, the following equation is obtained:

$$
\dot{U}(0)[T(t)+\tau \dot{T}(t)]=0
$$

From (16), it is obvious that $\dot{U}(0)=0$. Substituting $\dot{U}(0)=0$ into (11) gives $C_{2}=0$ and then $U(x)$ can be expressed by

$$
U(x)=C_{1} \cos \lambda x .
$$

Equation (17) presents the free axial vibration of the dense paste in pipeline.

Similarly, using $u(x, t)=U(x) T(t)$ in (7) gives

$$
\dot{U}(l)[T(t)+\tau \dot{T}(t)]=0 .
$$

Equations (17) and (18) lead to the following result:

$$
\sin \lambda l=0,
$$

where (19) is the circular frequency equation of free axial vibration of the paste rod in pipeline, and

$$
\lambda=\frac{n \pi}{l} .
$$

Then, $U(x)$ and $\omega$ can be obtained:

$$
\begin{aligned}
U(x) & =C_{1} \cos \frac{n \pi}{l} x \\
\omega & =\frac{n \pi}{l} c \sqrt{1-\frac{n^{2} \tau^{2} \pi^{2} c^{2}}{4 l^{2}}},
\end{aligned}
$$

where $n$ is the maximum integer less than $(2 l / \pi \tau c+1 / 2): n=$ $0,1,2,3, \ldots, m$.

For the superposition of $m$ main free vibration of dense paste, the free vibration equation can be written based on (11) and (12); the expression of the axial vibration displacement can be developed to

$$
\begin{aligned}
& u(x, t) \\
& =\sum_{n=1}^{m} e^{-\alpha t} \cos \left[\frac{n \pi}{l} x\right]\left[B_{1, n} \cos \left(\omega_{n} t\right)+B_{2, n} \sin \left(\omega_{n} t\right)\right] \\
& \quad+B_{1,0}+B_{2,0} t
\end{aligned}
$$

where $n$ means the undetermined coefficients which relates to the $n$ principal frequency; $m$ is the number of vibration orders. In (23) the coefficients $B_{1, n}, B_{2, n}, B_{1,0}$, and $B_{2,0}$ are constants according to the initial conditions, which are introduced later in Section 2.3. With (23), once the pressure from pump is absent, the free axial vibration of each microunit on the paste rod could be evaluated; the displacement of the units on the paste rod is able to be calculated. Based on the consideration that the reversed flow is the equivalent result of the backward moving of the microunits, it is reasonable to predict the percentage of reversed flow share according to the displacement of a reference microunit on the paste rod.

2.3. The Free Vibration of Initial Disturbance. While the pump pressure $f$ disappeared suddenly, which means there is no pressure imposing on the paste rod, so the initial axial displacement is

$$
u(x, 0)=\varepsilon(l-x)=\frac{F(l-x)}{E A}=\varphi(x) .
$$

At the initial time the velocity is zero, which is also presented by

$$
\dot{u}(x, 0)=0=g(x) .
$$

Introducing the initial displacement and velocity conditions, that is, (24) and (25), into the main vibration equation (23) gives the following expressions:

$$
\begin{aligned}
B_{1,0}+\sum_{n=1}^{m} B_{1, n} \cos \left[\frac{n \pi}{l} x\right] & =\frac{F(l-x)}{E A} \\
B_{2,0}+\sum_{n=1}^{m}\left(-\alpha B_{1, n}+B_{2, n} \omega_{n}\right) \cos \left[\frac{n \pi}{l} x\right] & =0 .
\end{aligned}
$$

Choosing the relaxation time $\tau$ as a smaller one, then the number of vibrations $m \rightarrow \infty$. Equation (26) can be considered as Fourier cosine series expansion of $\varphi(x)$ and $g(x)$ on the interval $[0, l]$ approximately:

$$
\begin{aligned}
& B_{1,0}=\frac{1}{l} \int_{0}^{l} \frac{F(l-x)}{E A} d x=\frac{F l}{2 E A} \\
& B_{1, n}=\frac{2 F l\left[1-(-1)^{n}\right]}{n^{2} \pi^{2} E A} \\
& B_{2,0}=0 \\
& B_{2, n}=\frac{\alpha}{\omega_{n}} \frac{2 F l\left[1-(-1)^{n}\right]}{n^{2} \pi^{2} E A} .
\end{aligned}
$$

The coefficients $B_{1, n}, B_{2, n}, B_{1,0}$, and $B_{2,0}$ can be calculated by (27), (28), (29), and (30), respectively. By introducing these coefficients back into (23), the vibration equation of paste rod when the pump pressure changed to zero suddenly is obtained eventually. 
TABLE 1: The first five principal frequencies of the slime rod.

\begin{tabular}{llllll}
\hline$L(\mathrm{~m})$ & $n=1$ & $n=2$ & $n=3$ & $n=4$ & $n=5$ \\
\hline$f(\mathrm{~Hz})$ & 0.705 & 1.410 & 2.224 & 2.819 & 3.524 \\
\hline
\end{tabular}

\section{Results and Discussion}

3.1. Reversed Flow Results by Axial Vibration Model. When dense pastes are transported in pipeline steadily during a certain piston travel, the axial pressure equals the radial pressure [14], which enables the pastes to be regarded as linear elastic body. The relationship amongst Young's modulus E, volumetric modulus of elasticity, and Poisson's ratio is as follows:

$$
E=3 K(1-2 v),
$$

where $K$ is the bulk modulus of elasticity of the paste and $v$ is Poisson's ratio.

Hao et al. [5] have calculated the true bulk modulus of coal slime in pipeline transportation. While the true bulk modulus of coal slime is $2.98 \mathrm{GPa}$, the pressure of the pump is $p=2 \mathrm{MPa}$ in the experiment. The coal slime consists of solid particle and liquid water and gives Poisson's ratio at 0.34 ; then Young's modulus $E=2.98 \mathrm{GPa}$ is obtained. As the concentration of the coal slime increases from 55\% to $75 \%$, the apparent viscosity increases from $25 \mathrm{~Pa} \cdot \mathrm{s}$ to $470 \mathrm{~Pa} \cdot \mathrm{s}$ [15].

By substituting the fixed value of parameters $(E, \eta, \rho$, $\pi, n$, and $l=1000 \mathrm{~m}$ ) into (22), the circular frequency of the axis vibration of the paste rod is obtained, from which the five principal frequencies are calculated as shown in Table 1 . The natural axial vibration frequency of the paste rod is $0.705 \mathrm{~Hz}$, and the first 5 orders are all below $3.524 \mathrm{~Hz}$.

It is obvious that $F=p A$, so (27), (28), and (30) can be written as

$$
\begin{aligned}
& B_{1,0}=\frac{P l}{2 E} \\
& B_{1, n}=\frac{2 P l\left[1-(-1)^{n}\right]}{n^{2} \pi^{2} E} \\
& B_{2, n}=\frac{\alpha}{\omega_{n}} \frac{2 P l\left[1-(-1)^{n}\right]}{n^{2} \pi^{2} E} .
\end{aligned}
$$

By substituting (32) into (23), the axial vibration displacement of coal slime paste in pipeline can be obtained. The axial vibration displacement curve for the microunit of the paste rod is drawn in Figure 4.

Figure 4 takes the displacement response of the unit at the left end $(x=0)$ of the coal slime rod, for example. Because of the driving by the pump, in certain time period, this unit moves to location $x=0.64 \mathrm{~m}$ along the pipe. The location from $x=0$ to $0.64 \mathrm{~m}$ is filled by new coal slime that pressed into the pipe by the pump. If this moment is set as the initial time $(t=0)$, the initial position of the concerned unit is therefore at $x=0.64 \mathrm{~m}$. In the process, the $S$-shape pipe valve stays for 0.7 minutes and then switches quickly in 0.7 seconds, which means 0.7 seconds' pressure pause. During this short moment, the concerned unit shifts from location $x=0.64 \mathrm{~m}$

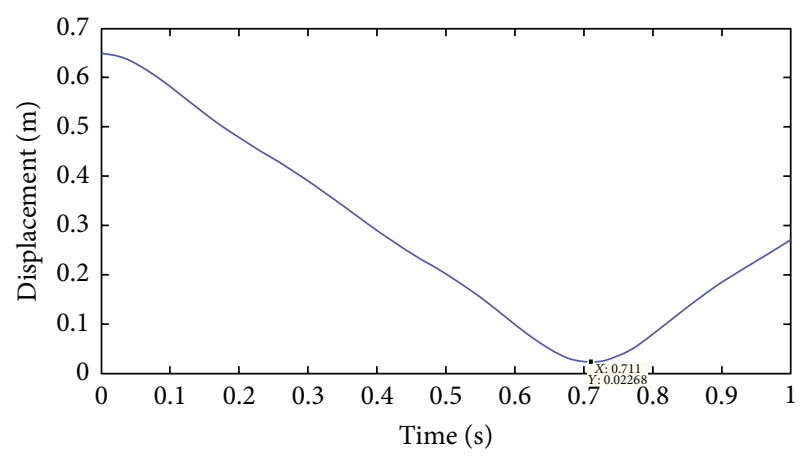

Figure 4: Displacement of the slurry unit at $x=0$ varies in one second.

to $0.03 \mathrm{~m}$. This traveling from $x=0.64 \mathrm{~m}$ to $0.03 \mathrm{~m}$ could be regarded as the length of the equivalent cylinder that was formed by the reversed flow. In other words, the theoretical length of the paste rod that contributes to the reversed flow is

$$
L=0.64 \mathrm{~m}-0.03 \mathrm{~m}=0.61 \mathrm{~m} .
$$

Apparently, the percentage of reversed flow share is equal to the ratio of the obtained displacement in (11) to the total length of the piston travel. The length of piston stroke is $1.20 \mathrm{~m}$; then

$$
\delta=\frac{L}{L_{0}}=\frac{0.61}{1.20}=50.8 \%
$$

The percentage of reversed flow volume would be different if the dense pastes or the transport conditions change. In order to identify the feasibility of the axial vibration model, the transport condition of slurry in pipeline is also analysed based on wave velocity method as explained in Section 3.2.

3.2. Estimation of Reversed Flow Share Based on Wave Velocity Method. In the pipeline transportation, the flow rate is considered to be the product of the discharged volume of each piston travel and the times of the piston travel in certain time:

$$
Q_{t}=\frac{n k \pi D_{1}^{2} L_{0}}{4}
$$

where $Q_{t}$ is the theoretical volumetric flow rate; $k$ is the efficiency of the pump; $D_{1}$ is the diameter of the pump. $L_{0}$ is the stroke length of piston and $n$ is the frequency of the stroke in given time.

How to calculate the reversed flow rate is introduced in detail in literature [5]. The following is concluded: if all the compressed paste in the pipe flows back to the feed chamber when the pressure from the pump disappears, the reversed flow rate could be calculated by the following equation:

$$
Q_{r}=\frac{n \pi D^{2}}{4} \int_{0}^{l} \frac{p}{K_{c}} d x
$$

where $Q_{r}$ is the volumetric reversed flow rate, $D$ is the diameter of the pipeline, $l$ is the length of pipeline, $K_{c}$ is the 
composite modulus of the fluid and the pipe, and $p$ is the pressure along the pipeline. $d x$ is the length of the microunit along the paste rod.

The study [5] showed that the pressure distribution along the pipeline can be expressed as (13):

$$
p=-2780 x+2.5 \times 10^{6}
$$

where $x$ is the distance from the exit of pump, given by $m$. According to the manual guide, the theoretical flow rate of the pump is $15.90 \mathrm{~m}^{3} / \mathrm{h}$; the calculated reversed flow volume is $9.64 \mathrm{~m}^{3} / \mathrm{h}$ in the experiment. Therefore the percentage of reversed flow volume is

$$
\delta=\frac{Q_{r}}{Q_{t}}=\frac{9.64}{15.90}=60.6 \% .
$$

This value in (38) which was calculated by bulk elasticity analysis is close to the result based on axial vibration, which is presented in (34).

The two methods to estimate reversed flow volume are both feasible in the application of the dense paste in pipeline, while the concepts and the used parameters are very different if the samples and the experiment system change from one situation to another and if researchers want to study all situations amounts of experiments are needed; this vibration model is to simplify the experimental process.

Although the two results have small difference in between, considering the fact that the results are obtained in the engineering field, they can be accepted and they can satisfy the needs of the engineering. And there are many factors which affected the testing results, such as the slope of the pipeline and the uniformity of the material. In addition, there exists an error in the wave velocity measurement process, for example, the displacement sensor measure error and the cylinder volume change measurement including paste volume, deformation, and paste test cylinder leaks. Since both methods are based on numerical estimation and the real reversed flow rate is hard to measure exactly in experiments, it is believed that the calculation based on axial vibration model is still applicative for coal slime in pipeline transportation projects.

\section{Conclusions}

According to the fact that the flowing dense paste in pipeline behaves as the plug flow, together with the phenomenon that the dense paste under pressure shows the character of viscoelasticity, a proposal to treat the body of the dense paste in the straight pipe as a viscoelastic rod was carried out to analyze the axial vibration of the paste and the reversed flow during transportation. The axial vibration model of the rod is formulated based on the force balance of the flowing rod in the pipe. Then, initial conditions for the axial vibration were refined according to the working situations of the pumps in the pipeline transportation system, leading to the solved terms about the free axial vibration of the rod, including the vibration model function, the displacement of the microunits on the rod, and the primary vibration frequencies in orders. This work by introducing the axial vibration model into the analysis of the vibration intensity of the paste body and its influence on the long-distance pipeline transportation system is distinctive compared to other similar researches in this field.

In a further step, using the results about the displacement of the microunit on the paste rod during vibration, the displacement response was plotted, from which the relationship between the vibration and the resulting reversed flow was deduced numerically and simply. By taking the instance that coal slime pastes are transported in pipelines, the reversed flow share was estimated to be $50.8 \%$, which in the engineering application is considered to be close to $60.6 \%$, which was obtained based on wave velocity method in pipeline transportation.

Additionally, some relative conclusions during the analysis of axial vibration of the coal slime paste rod are summarized as follows:

(1) The nature vibration frequencies of the coal slime paste rod are very low. The elastic recovery of the compressed paste, which is modeled as one process of the vibration of the viscoelastic rod in this paper, mainly forms the reversed flow from the pipe to the chamber of the pump.

(2) The displacement of the rod during the vibration physically depends on the elasticity modulus, viscosity, density of the paste, and the transportation conditions like the distance, the rated pumping pressure, and so on.

(3) In the application case of transporting coal slime paste, the impact on the axial vibration from the apparent viscosity of the coal slime paste is negligible; the vibration displacement of the material at the exit of the pump decreases as the elasticity modulus of the paste increases; when lengthening the transportation distance of the system, the corresponding vibration displacement of the material at the exit of the pump and the average displacement of the whole coal slime paste rod rise at the same scale.

\section{Competing Interests}

The authors declare that there are no competing interests regarding the publication of this paper.

\section{Acknowledgments}

This work is supported by the national natural science fund project (51075389 and 51406106) and Research Fund for the Doctoral Program of Higher Education of China (20100023110006).

\section{References}

[1] A. Fourie, "Paste and thickened tailings: has the promise been fulfilled?" in Proceedings of the GeoCongress, pp. 4126-4134, Oakland, Calif, USA, March 2012. 
[2] R. Jewell, "The evolution of paste and thickened tailings," Australian Center for Geomechanics Newsletter, vol. 35, pp. 2-3, 2000.

[3] A. J. C. Paterson, "High density slurry and paste tailings, transport systems," in Proceedings of the International Platinum Conference Platinum Adding Value, pp. 159-165, Sun City, South Africa, October 2004.

[4] T. Belem and M. Benzaazoua, "An overview on the use of paste backfill technology as a ground support method in cut-andfill mines," in Proceedings of the 5th International Symposium on Ground Support in Mining and Underground Construction, Perth, Australia, September 2004.

[5] X. Hao, N. Li, X. Jia, S. Zhang, and M. Wu, "Measurement of bulk modulus of elasticity of dense pastes and its effects on flow rate in long pipeline," Minerals Engineering, vol. 49, pp. 145-153, 2013.

[6] M. Wu, X. D. Hao, W. Jing, and D. K. Zhu, "Pipeline transport and utilization of coal sludge," in Proceedings of the International Coal Preparation Congress, Lexington, Ky, USA, April 2010.

[7] Z. Lidian, "Analysis of pressure pipeline vibration causes," Pipeline Technique and Equipment, no. 2, pp. 31-32, 2006.

[8] X. Liang and Y. Zhao, "Wavelet analysis on vibration signal of service pipe and study on vibration elimination," China Safety Science Journal, no. 2, pp. 70-75, 2005.

[9] Z.-Y. Xia, Z.-H. Zhang, B.-Z. Wang, Y. Huang, Y. Guo, and J. Wang, "Research on theoretical calculation method of natural frequency of the pendulum wave power conversion device," Ocean Technology, vol. 30, no. 1, pp. 91-94, 2011.

[10] Z. Yanzhi, C. Nan, and X. Baoliang, "Free vibration analysis of viscoelastic foundation plate on viscoelastic foundation," Structure \& Environment Engineering, no. 3, pp. 31-41, 2001.

[11] Y. Zhu, B. Xue, and G. Wang, "Free vibration analysis of viscoelastic foundtion plate on viscofiastic foundtion," Chinese Journal of Rock Mechanics and Engineering, vol. 21, no. 1, pp. 112118, 2002.

[12] L. Ting, "Analytical solutions of stress wave propagation in viscoelastic pile," Journal of Vibration and Shock, vol. 19, no. 4, pp. 10-12, 2000.

[13] L. Fuyan, J. Xuankai, L. Yayun, X. Zhengmeng, H. Xuedi, and W. Miao, "Experimental study on pressure impact and vibration of long-distance coal slime transportation pipeline," Industry and Mine Automation, vol. 41, no. 5, pp. 91-94, 2015.

[14] Z. Wang, Research on pressure transmission characteristics of dense pastes during pipeline transportation [Ph.D. thesis], China University of Mining \& Technology, Beijing, China, 2013.

[15] X. Y. Zhao, Experimental study on classification method and pipeline transport properties of high concentration and viscous materials [Ph.D. thesis], China University of Mining \& Technology, Beijing, China, 2005. 


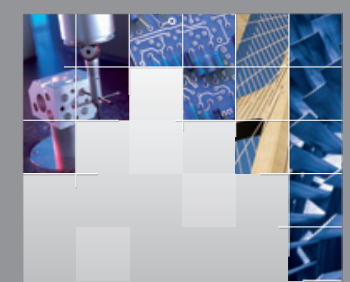

\section{Enfincering}
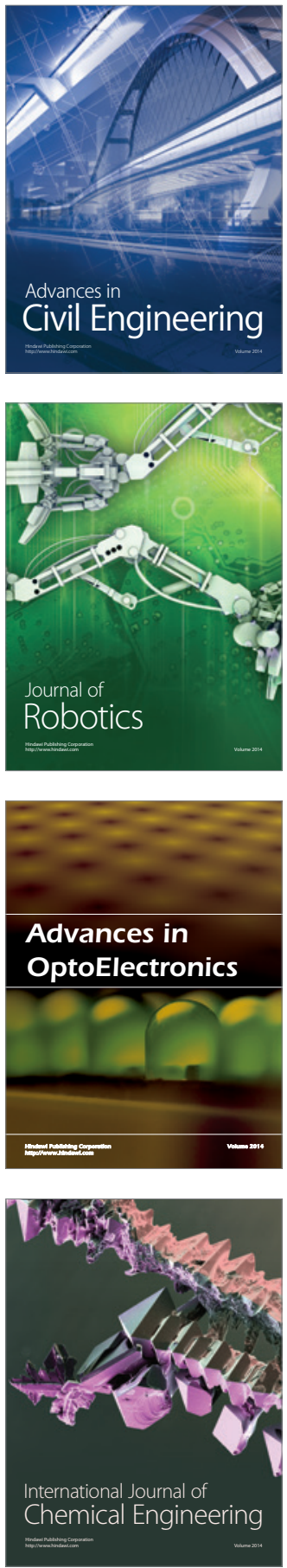

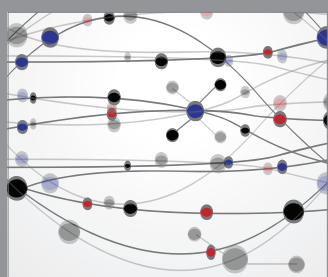

The Scientific World Journal

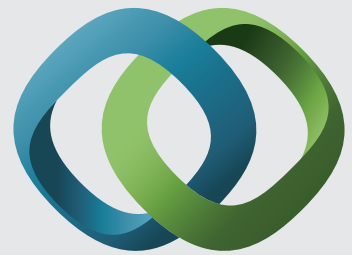

\section{Hindawi}

Submit your manuscripts at

http://www.hindawi.com
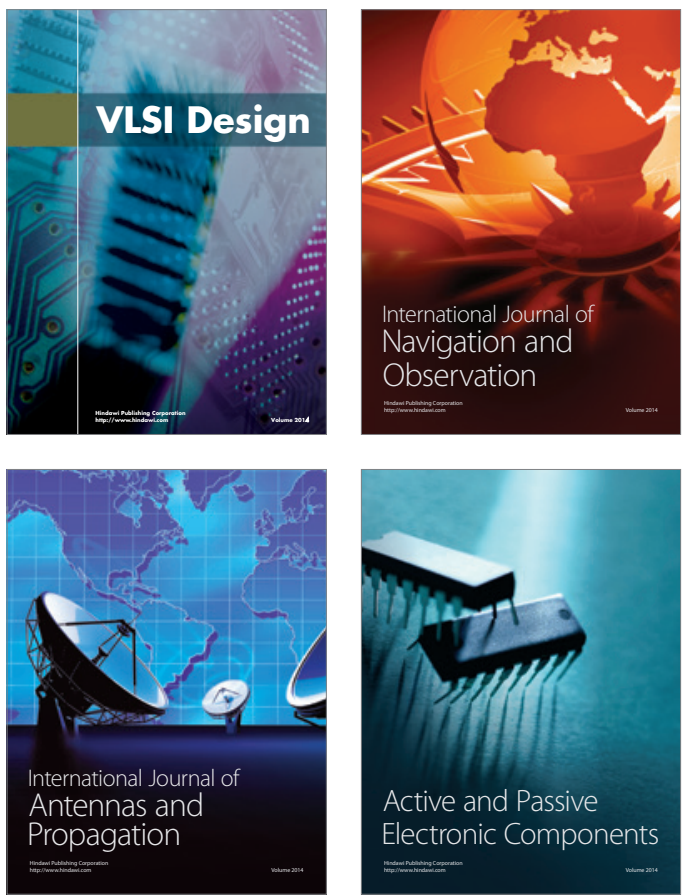
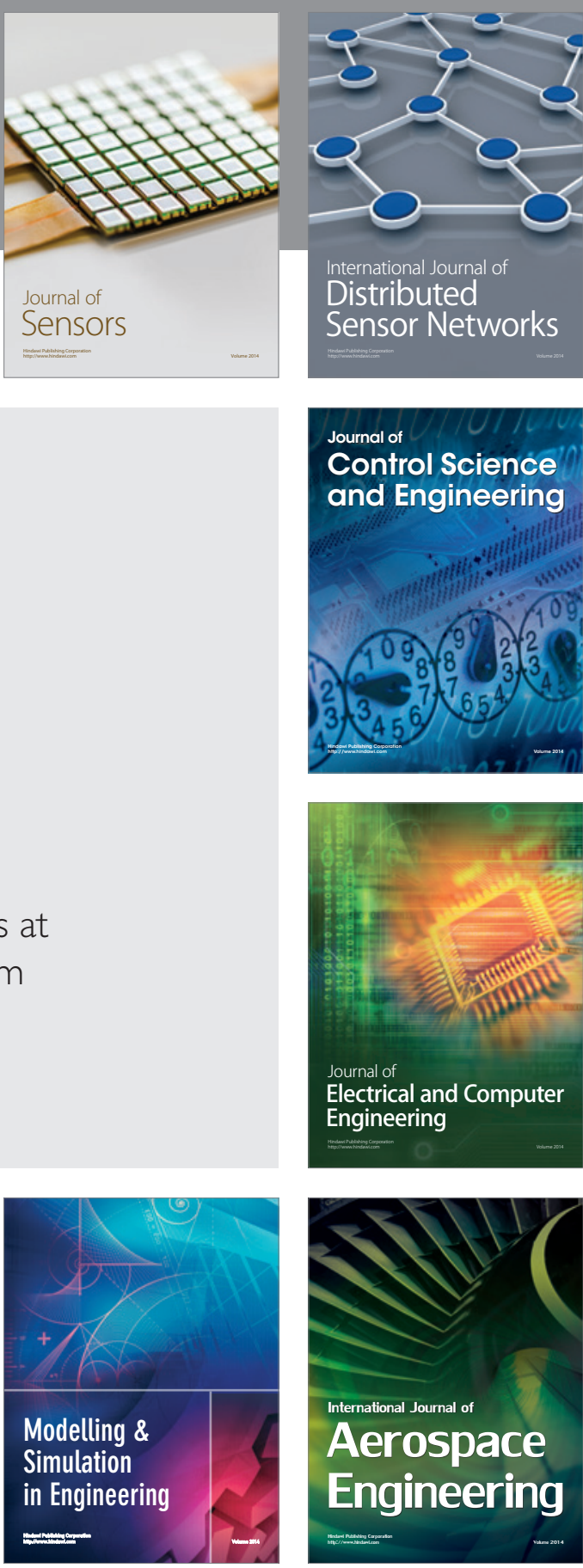

International Journal of

Distributed

Sensor Networks

Journal of

Control Science

and Engineering
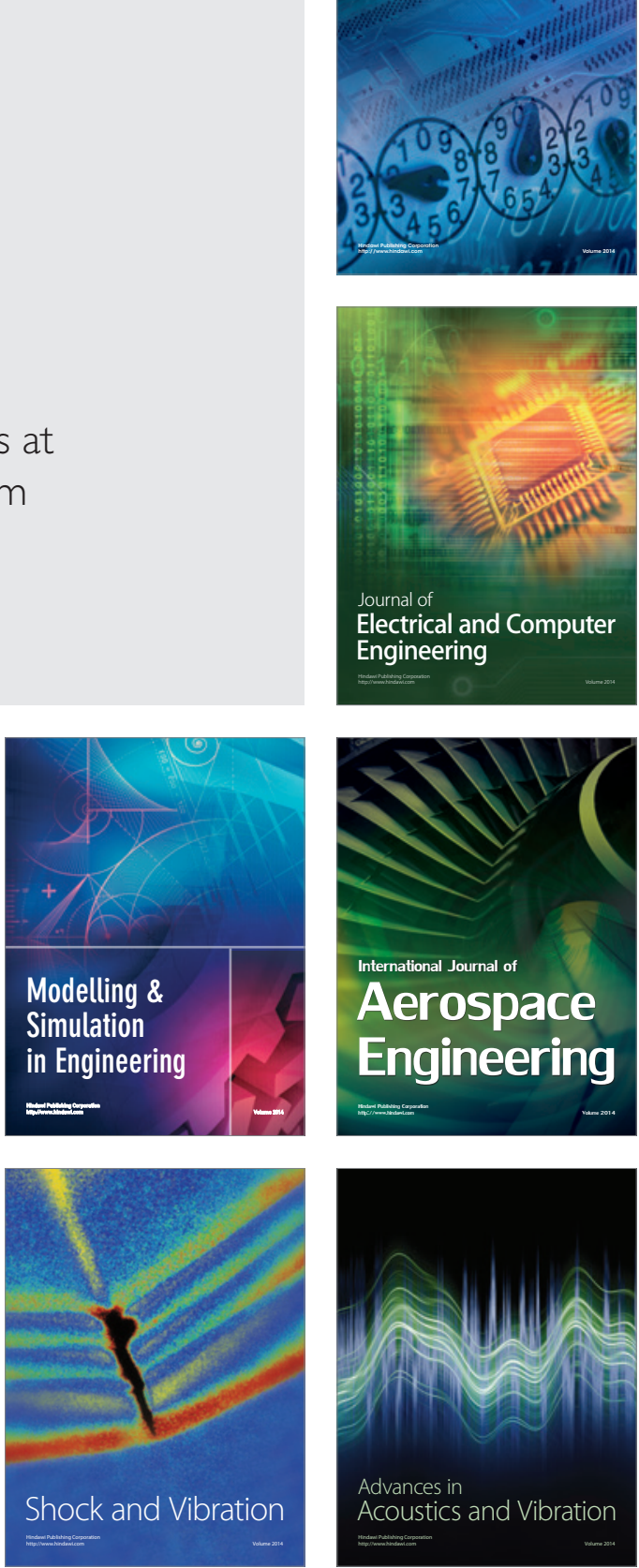